\title{
FORMAÇÃO E CARACTERIZAÇÃO ÓPTICA DE FILMES AUTOMONTADOS DE POMA/PPV
}

\author{
Rodrigo M. Melo e Noelio O. Dantas
}

Faculdade de Física, Universidade Federal de Uberlândia, CP 593, 38400-902 Uberlândia - MG

Nara C. de Souza, Osvaldo N. Oliveira Jr., Roberto M. Faria e Alexandre Marletta*\#

Instituto de Física de São Carlos, Universidade de São Paulo, CP 369, 13560-970 São Carlos - SP

Recebido em 5/2/02; aceito em 5/7/02

\begin{abstract}
FORMATION AND OPTICAL CHARACTERIZATION OF POMA/PPV SELF-ASSEMBLY FILMS. A study is presented of the formation and optical properties of polymeric heterostructures from poly( $p$-phenylene vinylene) (PPV) and poly $(o$-methoxyaniline) (POMA) produced via the self-assembly technique. POMA layers were obtained in a non self-limiting process from its emeraldine salt, semiconducting form in $\mathrm{HCl}$ solution. Thermal conversion of PPV was performed at low temperatures with the substitution of the counter-ion $\mathrm{Cl}$ in the PPV precursor by a long sulfonic chain, the dodecylbenzenesulfonate (DBS) ion. The optical properties of PPV films converted in this way are not affected by POMA, which can be used as transparent electrode of PPV luminescent devices.
\end{abstract}

Keywords: poly( $p$-phenylene vinylene); poly (o-methoxyaniline); heterostructures.

\section{INTRODUÇÃO}

Os polímeros conjugados e emissores de luz $z^{1}$ apresentam propriedades ópticas e eletrônicas similares às dos semicondutores inorgânicos. Apresentam a vantagem, porém, de serem produzidos na forma de filmes finos e ultrafinos a um baixo custo, o que os tornam interessantes tanto do ponto de vista tecnológico (eletrônica de filmes finos), quanto da física fundamental (estrutura eletrônica). Suas propriedades físicas dependem, essencialmente, da estrutura química dos monômeros, que constituem a cadeia principal polimérica, podendo alterar a região espectral de emissão ${ }^{1}$. Há, atualmente, dispositivos eletrônicos utilizando esses materiais orgânicos, como transistores de efeito de campo, diodos eletroluminescentes e dispositivos fotocondutores ${ }^{2}$. Dentro deste contexto, um dos polímeros conjugados mais promissores é o poli( $p$-fenileno vinileno) (PPV) ((2) na Figura 1), obtido a partir do processamento e da conversão térmica de um polímero precursor não conjugado, o poli(cloreto de xilideno tetrahidrotiofeno) (PTHT) ((1) na Figura 1). A formação de filmes de PPV só foi possível devido a progressos significativos na síntese e processabilidade deste material ${ }^{3}$.

Convencionalmente, o processo de conversão térmica $\left(\mathrm{T}_{\text {conv }}\right)$ do PPV, em que ocorre a eliminação dos grupos laterais sulfonados do PTHT, é realizado a temperaturas altas $\left(\sim 250^{\circ} \mathrm{C}\right)$ durante longos tempos $(\sim 6 \mathrm{~h})$ em vácuo (reação (i) na Figura 1). Entretanto, este procedimento não é compatível com as etapas de processamento de dispositivos à base de PPV, envolvendo múltiplas camadas poliméricas, em que são utilizados materiais com propriedades físicas, tais como pontos de fusão e condutividade, distintas ${ }^{4}$. Uma rota alternativa é a conversão à baixa temperatura, desenvolvida em nossos laboratórios, que consiste em substituir o contra-íon do polímero precursor pelo íon dodecilbenzenosulfonato (DBS $)^{5}$, como indicado pela reação (ii) na Figura 1. O resultado final é um polímero precursor substituído do PPV apresentado no esquema (3) (Figura 1). A conversão térmica do PPV, utilizando este procedimento, é realizada em um tempo característico de apenas $30 \mathrm{~min}$

*e-mail: marletta@ufu.br

\# endereço atual: Faculdade de Física, Universidade Federal de Uberlândia, CP 593, 38400-902 Uberlândia - MG

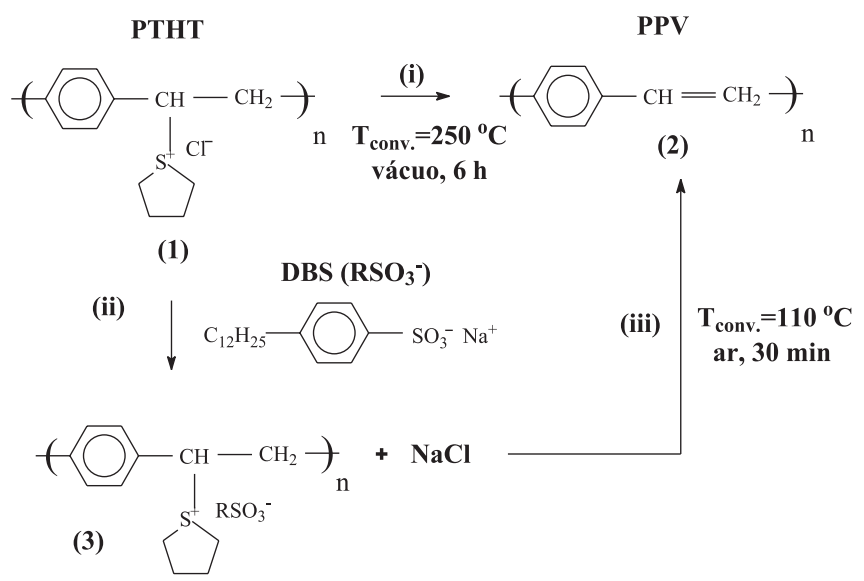

Figura 1. Esquema do processo de conversão do PPV à baixa temperatura: (i) esquema da rota convencional de tratamento térmico para obter PPV; (ii) adição do DBS ao PTHT; (iii) processo de eliminação do grupo de saída tetrahidrotiofeno complexado com o contra-íon DBS a baixas temperaturas $\left(110^{\circ} \mathrm{C}\right)$ em tempos rápidos $(30 \mathrm{~min})^{5}$

sob pressão ambiente e a $110^{\circ} \mathrm{C}$, como mostra a reação (iii) na Figura 1 . Esta reação de termo eliminação completa-se em $2 \mathrm{~h}$. Resultados de filmes "spin-coated" de PPV+DBS indicaram que tal tratamento térmico não compromete as propriedades ópticas do filme ${ }^{5}$. Abre-se, então, a possibilidade de se usar o PPV como camada ativa, com uma maior eficiência, devido a menor geração de defeitos estruturais (por exemplo, incorporação do grupo carbonila) e a utilização de outros materiais poliméricos de baixo ponto de fusão.

A importância tecnológica da combinação de vários materiais poliméricos, por exemplo, formando um sistema de múltiplas camadas, é relatada por Yang e Heeger ${ }^{6}$. A simples combinação de óxido de estanho/índio (ITO) e polianilina (PAni), como ânodo transparente, e o poli(2-metoxi-5-(2'-etilexiloxi)-1,4-fenileno vinileno) (MEH-PPV) promoveu um aumento da performance dos diodos emissores de luz (LEDs) de ITO/PAni/MEH-PPV/Ca de 30-40\% e 
uma diminuição na voltagem de operação de 30-50\%. A PAni também foi utilizada em estruturas ITO/PAni/PPV+DBS/Al ${ }^{7}$, em que se aproveitou o processo de conversão rápida do PPV a baixas temperaturas $^{5}$. Este sistema apresentou uma diminuição na tensão de operação dos LEDs em cerca de 30\%, ilustrando a importância da utilização de vários materiais em aplicações tecnológicas reais. Entretanto, este simples exemplo de heteroestrutura não pode ser empregado ao PPV obtido convencionalmente, uma vez que materiais como a PAni têm sua estrutura e condutividade elétrica fortemente afetadas com tratamentos térmicos acima de $100{ }^{\circ} \mathrm{C}^{8}$.

Neste trabalho, apresenta-se um estudo das propriedades ópticas de filmes de PPV+DBS sobre uma camada de poli(o-metoxianilina) (POMA), ambos preparados pela técnica de automontagem. Os filmes de PPV foram obtidos após conversão térmica a alta $\left(230^{\circ} \mathrm{C}\right)$ e a baixa $\left(110{ }^{\circ} \mathrm{C}\right)$ temperatura. As caracterizações ópticas iniciais mostram a viabilidade na produção de heteroestruturas simples, com alta estabilidade estrutural da camada de POMA, quando o PPV é obtido a temperatura mais baixa.

\section{PARTE EXPERIMENTAL}

A poli(o-metoxianilina) é um polímero que em um dos seus estados de semi-oxidação, base de esmeraldina (Figura 2), pode ser protonado quando colocado num meio ácido. Nestas condições, a sua condutividade pode aumentar em até 11 ordens de grandeza (1 S.cm ${ }^{-1}$ ), isto é, torna-se um condutor elétrico ${ }^{9}$. A Figura 3 representa a POMA na forma de sal de esmeraldina. Essa forma é solúvel em água, podendo ser usada como solução catiônica. A carga elétrica deste polímero é dependente do pH da solução, o que faz com que ele seja um polieletrólito fraco. A POMA utilizada neste trabalho foi obtida na forma sal de esmeraldina, forma condutora de acordo com a rota descrita por Mattoso et al. ${ }^{10}$. A solução de POMA foi preparada dissolvendo-se o polímero $(\sim 1,202 \mathrm{~g})$ em acetonitrila e água ultrapura (Milli-Q), numa proporção de $20 \mathrm{~mL}: 980 \mathrm{~mL}$. Esta solução permaneceu sob agitação magnética à temperatura ambiente por $4 \mathrm{~h}$. É necessário que exista um intervalo de tempo suficiente para que a solução, após agitação, permaneça em repouso a fim de "separar" da solução a fração do polímero, que não foi totalmente dissolvida. A solução é, então, submetida a um processo de filtragem, em um filtro cerâmico com porosidade de 25 a $50 \mu \mathrm{m}$. A concentração desta solução foi de 0,6 g.L $\mathrm{L}^{-1}$.

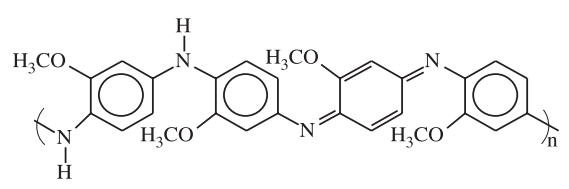

Figura 2. Poli(o-metoxianilina) como base de esmeraldina (desprotonada)

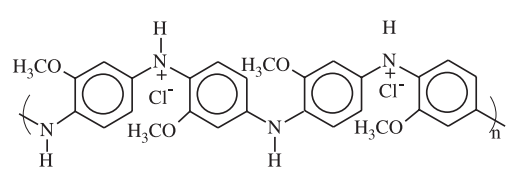

Figura 3. Poli(o-metoxianilina) como sal de esmeraldina (protonada)

Os filmes foram depositados sobre lâminas de BK7, utilizando o método de automontagem ${ }^{11}$. O tratamento aplicado, para hidrofilização das lâminas, teve início com sua exaustiva lavagem em água e detergente. Uma solução de peróxido de hidrogênio $\left(\mathrm{H}_{2} \mathrm{O}_{2}\right)$, com ácido sulfúrico $\left(\mathrm{H}_{2} \mathrm{SO}_{4}\right)$ numa proporção 3:7, foi utilizada para o tratamento das lâminas. O béquer, contendo esta solução juntamente com as lâminas, foi mantido em ultra-som durante $1 \mathrm{~h}$. Os substratos foram retirados e novamente lavados com água ultrapura (Milli-Q). Após a lavagem em água ultrapura, as lâminas foram submetidas a uma nova solução contendo água (Milli Q), peróxido de hidrogênio $\left(\mathrm{H}_{2} \mathrm{O}_{2}\right)$ e hidróxido de amônio $\left(\mathrm{NH}_{4} \mathrm{OH}\right)$, em razão volumétrica de $5: 1: 1$, respectivamente. Novamente o béquer, com a nova solução e as lâminas, foi mantido em ultra-som por $30 \mathrm{~min}$. As lâminas foram enxaguadas com água (Milli Q) até a completa remoção da solução de tratamento.

Filmes de 10 e 20 camadas foram obtidos a partir da imersão, por 2 min, do substrato em solução de POMA e posterior secagem em jato de $\mathrm{N}_{2}$. Note que as camadas de POMA não foram alternadas com um polímero aniônico, como é usual. A adsorção de uma camada de POMA sobre POMA já depositada, é possível, ainda assim, porque há fortes interações com ligações de hidrogênio ${ }^{12}$, responsáveis por uma adsorção não autolimitada ${ }^{13}$. A concentração do policátion precursor do PPV (PTHT) foi de $0,3 \mathrm{mg} \cdot \mathrm{mL}^{-1}$. A solução de DBS foi preparada em água Milli-Q numa concentração de $0,1 \mathrm{M}$ e pH = 5,0. Os tempos de imersão das lâminas de BK7, contendo a POMA nas soluções de PTHT e DBS, foram de 30 seg em cada uma, sendo os substratos secos com jato de $\mathrm{N}_{2}$, formando 20 camadas de PTHT+DBS. O resultado final desta deposição está representado esquematicamente na Figura 4. A camada de POMA tem um excesso de carga iônica positiva, devido à sua forma de sal esmeraldina. As camadas de PTHT+DBS são incorporadas subseqüentemente. É importante ressaltar que o DBS é incorporado como contraíon do grupo tetrahidrotiofeno (esquema (3) na Figura 1). Finalmente, os filmes foram submetidos à conversão térmica a $110{ }^{\circ} \mathrm{C}$ e a $230{ }^{\circ} \mathrm{C}$, em vácuo $\left(10^{-2} \mathrm{~atm}\right)$, por $2 \mathrm{~h}$, para obter o PPV.

As medidas de absorbância no UV-Vis foram realizadas em um espectrofotômetro Hitachi U-2001. Os espectros de luminescência foram obtidos com a seguinte montagem experimental: um Laser de $\mathrm{Ar}^{+}$excitando a amostra em $458 \mathrm{~nm}$, a emissão analisada por um monocromador DIGIKROM DK480 e detectada por uma fotomultiplicadora tipo Hamamatsu R446. As amostras foram acondicionadas em um criostato de circuito de hélio, fechado sob vácuo de $10^{-5}$ torr.

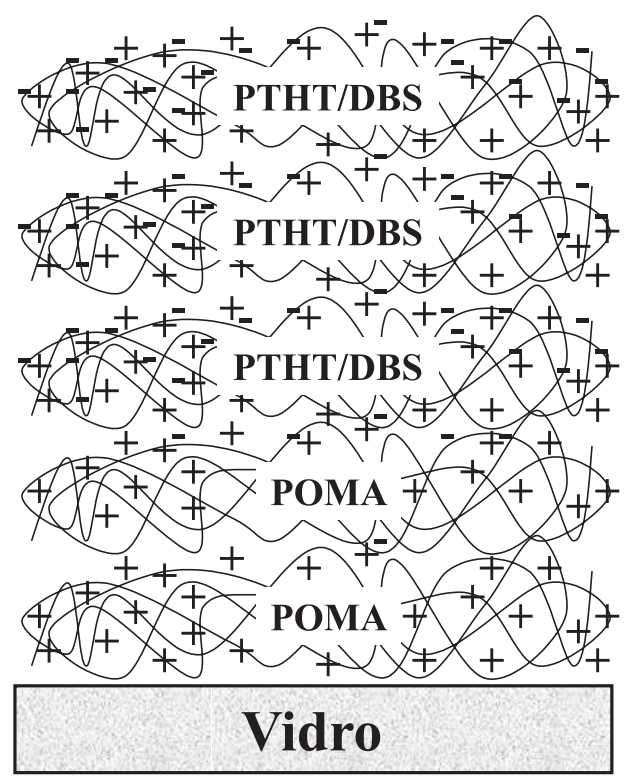

Figura 4. Esquema do método de automontagem, camadas de PTHT+DBS sendo adsorvidas sobre o filme de POMA 


\section{RESULTADOS E DISCUSSÃO}

O número de vezes que o substrato é imerso na solução de POMA, e seguidamente seco em fluxo de nitrogênio, tem efeito na cinética de adsorção. Isso pode ser visto na Figura 5, que representa a absorbância máxima versus o número de camadas, para um dado período de imersão na solução de POMA. Da análise da curva observa-se que, quanto maior o tempo (2 min) de imersão e secagem do substrato, maior é a quantidade de POMA adsorvida. Por outro lado, sabe-se que cada vez que o substrato é retirado da solução, interrompe-se o processo termodinâmico de adsorção. Este aumento da quantidade adsorvida pode ser explicado analisando-se o que ocorre quando da imersão do substrato hidrofilizado na solução de POMA. Tanto moléculas de POMA como moléculas de água podem ser adsorvidas. Quando se retira o substrato da solução e o seca, as moléculas de água adsorvidas se desprendem e novos sítios ativos são criados. Quando o substrato retorna à solução, as regiões anteriormente preenchidas com água voltam a ser disputadas por novas moléculas (água e POMA). Portanto, quanto mais vezes se seca o substrato, mais ele adsorve polímero. Essa curva de cinética de adsorção apresentou um crescimento característico diferente, que foi primeiro detectado por Raposo $^{12}$ e investigado posteriormente por Pontes ${ }^{13}$, em que ao invés de ocorrer saturação na adsorção, após um tempo característico, esta continuava a crescer enquanto o processo fosse repetido. A esse comportamento os autores deram o nome de adsorção não autolimitada.

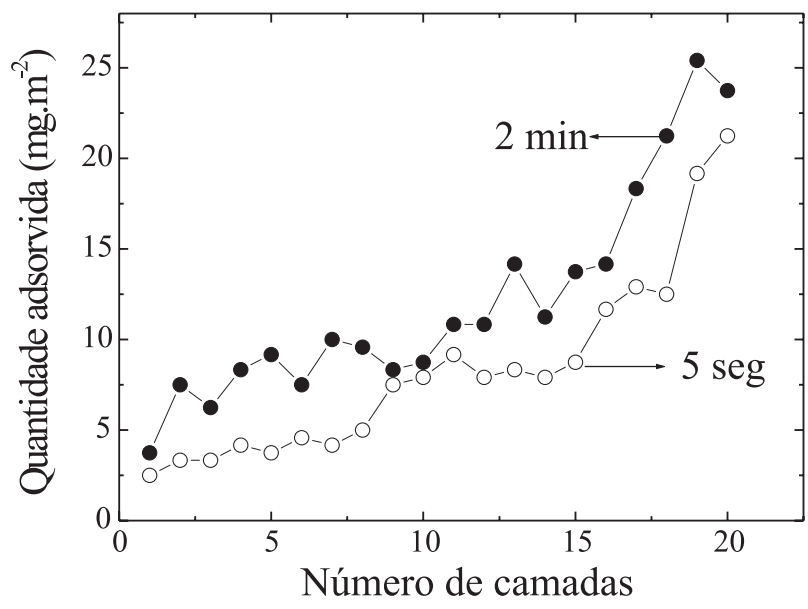

Figura 5. Absorbância máxima versus o tempo de imersão acumulado. A concentração da solução de POMA foi de $0,6 \mathrm{gL}^{-1}$ e o $\mathrm{pH}=3$

A utilização da POMA como possível eletrodo transparente em LEDs é demonstrada pelas propriedades ópticas ilustradas na Figura 6. Nota-se que entre 450 e $700 \mathrm{~nm}$ o filme automontado de 20 camadas de POMA com espessura de 3,6 nm praticamente não apresenta absorção, formando uma "janela" para a emissão do PPV, cujo espectro de fotoluminescência também aparece na figura para um filme "spin-coated" convertido a $230^{\circ} \mathrm{C}$, por $2 \mathrm{~h}$ sob vácuo. Para efeito de comparação, são mostrados os espectros de absorção de um filme de $180 \mathrm{~nm}$ de espessura de PAni obtida por deposição in situ ${ }^{7,8}$ e a emissão de um filme "spin-coated" de MH-PPV (poli-5-hexiloxi-2metoxi-p-fenileno vinilideno). A janela óptica da PAni, de 475 a 600 $\mathrm{nm}$, é mais estreita que a da POMA, superpondo a região de menor energia do espectro de emissão do PPV. Para o caso do MH-PPV, esta superposição torna-se mais significativa, em que deve ser esperada uma absorção pela camada de PAni. O espectro de PL do MEHPPV, utilizado por Yang ${ }^{6}$, é bastante semelhante em forma e posição energética ao do MH-PPV (Figura 6), mostrando que a PAni não é um eletrodo totalmente transparente no sistema PAni/MEH-PPV. Já no caso do sistema POMA/PPV, o eletrodo formado pela POMA é totalmente transparente, o que é essencial quando se objetiva maior eficiência de emissão em dispositivos eletroluminescentes. Mencione-se que a diferença fundamental entre as absorções da PAni e POMA, mostradas na Figura 6, está no fato de que a POMA apresenta energia de "gap" menor que a da Pani, quando estes materiais são dopados quimicamente. A POMA apresenta um pico de absorção em torno de $850 \mathrm{~nm}$, característico do estado dopado, e praticamente não tem absorção na região de $600 \mathrm{~nm}$.

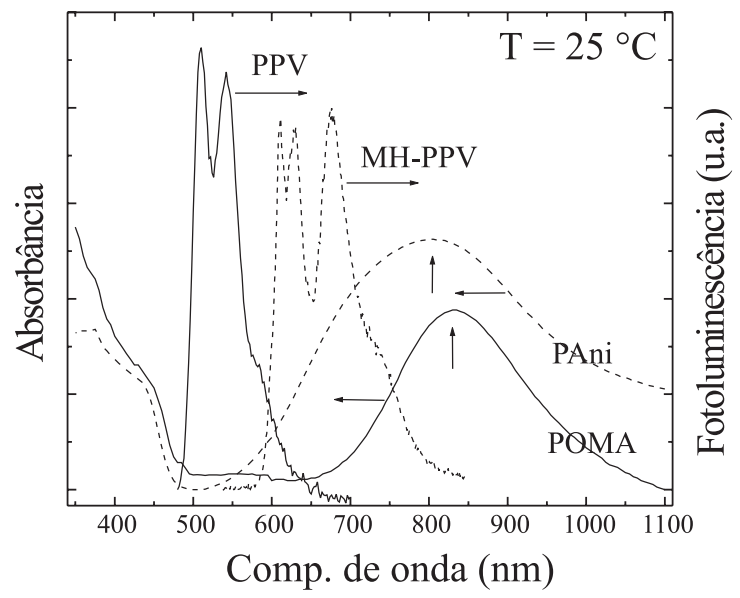

Figura 6. Espectro de absorbância na região do UV-Vis de um filme automontado de 20 camadas de POMA e espectro de fotoluminescência para um filme "spin-coated" de PPV convertido a $230{ }^{\circ} \mathrm{C}$ por 2 h sob vácuo (linhas contínuas). Para comparação (linhas tracejadas) é mostrado o espectro de absorbância de um filme de PAni depositado in situ $^{7,8}$ e o espectro de emissão de um filme "spin-coated" de MH-PPV

Na Figura 7 é apresentado o espectro de absorbância na região espectral do UV-Vis, para filmes de 20 camadas de PPV sobre POMA, convertido termicamente $\mathrm{a} \mathrm{T}_{\text {conv }}=110^{\circ} \mathrm{C} \mathrm{e} \mathrm{T}_{\text {conv }}=230{ }^{\circ} \mathrm{C}$ por $2 \mathrm{~h} \mathrm{sob}$ vácuo. Os filmes POMA foram produzidos com 10 camadas (Figura 7a) e 20 camadas (Figura 7b).

O aumento da temperatura de tratamento térmico dos filmes de POMA/PPV produz um deslocamento para o ultravioleta da banda

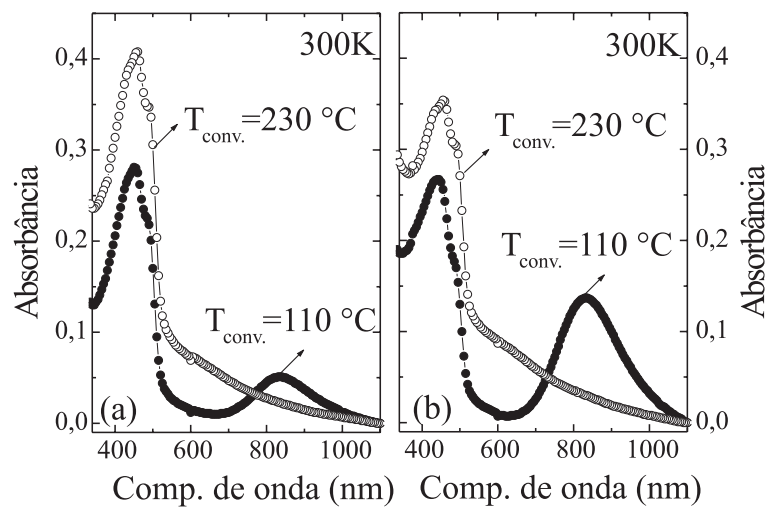

Figura 7. Espectros de absorbância na região do UV-Vis de filmes automontados de PPV+DBS de 20 camadas convertidos termicamente a $110{ }^{\circ} \mathrm{C}$ e $230{ }^{\circ} \mathrm{C}$ por $2 \mathrm{~h}$ sob vácuo e depositados sobre filmes de POMA de (a) 10 e (b) 20 camadas 
eletrônica $\pi-\pi^{*}$ (máximo em $875 \mathrm{~nm}$ ) da POMA, devido a uma desdopagem parcial dos filmes, similar ao que ocorre em PAni ${ }^{14,15}$. Além disso, para os filmes convertidos a $230{ }^{\circ} \mathrm{C}$ observa-se um aumento significativo da absorção entre 475 a 700 nm, devido à POMA, eliminando a janela para emissão do PPV. Por outro lado, a janela óptica se mantém para o filme convertido a $110^{\circ} \mathrm{C}$, com absorção desprezível na região de emissão do PPV. Nos espectros aparece, ainda, a banda de absorção do PPV entre 350 e $500 \mathrm{~nm}$, correspondente às transições eletrônicas dos estados não localizados da banda $\pi-\pi^{*}$ desse material. Em ambos os casos $\left(110^{\circ} \mathrm{C}\right.$ e $\left.230^{\circ} \mathrm{C}\right)$ nota-se que não há mudanças significativas na forma e posição da banda de absorção do PPV, demonstrando a eficiência do processo de conversão rápida à baixa temperatura, com a introdução do $\mathrm{DBS}^{5}$.

Na Figura 8 são apresentados os espectros de fotoluminescência (PL) para dois filmes, contendo 10 camadas de POMA e 20 camadas de PPV+DBS, convertidos termicamente a 110 e $230^{\circ} \mathrm{C}$. A Figura 8 mostra, também, a arquitetura do filme, simulando a estrutura de um LED, cuja luz é captada no lado do substrato.

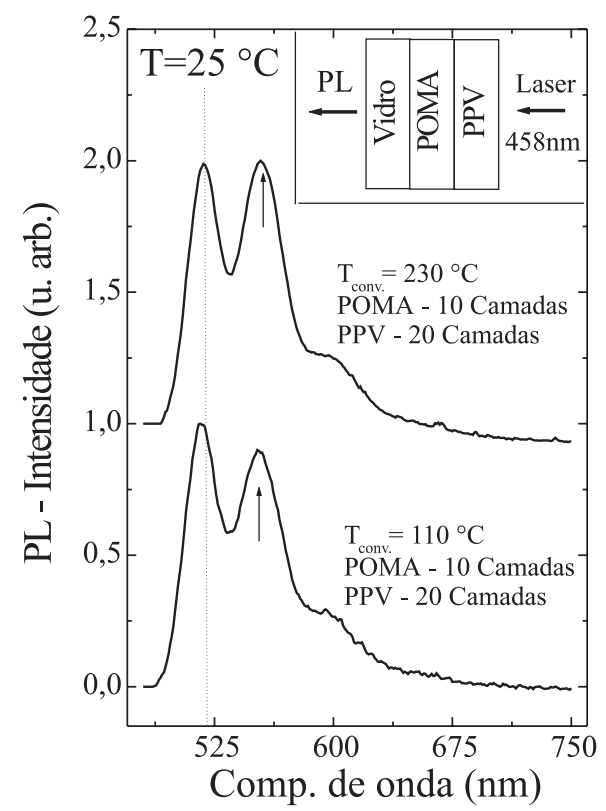

Figura 8. Comparação dos espectros de fotoluminescência de filmes de $P O M A / P P V+D B S$ (10 camadas de POMA e 20 camadas de PPV+DBS), convertidos termicamente a $110^{\circ} \mathrm{C}$ e $230{ }^{\circ} \mathrm{C}$

Observa-se que os espectros de PL possuem as mesmas estruturas, entretanto para o filme tratado a mais alta temperatura $\left(230^{\circ} \mathrm{C}\right)$, há um pequeno deslocamento para o vermelho $(2 \mathrm{~nm})$ e um aumento da intensidade espectral na região vibracional (indicado pelas setas). Este deslocamento é explicado pelo aumento do grau de conjugação efetivo ao longo das cadeias de PPV, uma vez que, a esta temperatura de conversão, há eliminação térmica do grupo lateral tetraidrotiofeno complexado com o contraíon $\mathrm{Cl}^{5}$. $\mathrm{O}$ aumento da primeira réplica de fônon (setas) indica o aumento da geração de defeitos estruturais com a criação, por exemplo, de grupos carbonila $(\mathrm{C}=\mathrm{O})$, devido à termo-oxidação que ocorre acima de $150{ }^{\circ} \mathrm{C}$.

\section{CONCLUSÕES}

Os resultados apresentados aqui demonstraram a possibilidade de produção de heteroestruturas luminescentes de PPV e POMA, utilizando-se a técnica de automontagem. As caracterizações ópticas dos filmes POMA/PPV mostram a possibilidade de utilização da POMA, como janela óptica para o PPV, com resultados melhores que os encontrados utilizando a PAni. A possibilidade de produção de dispositivos eletroluminescentes de múltiplas camadas, utilizando o PPV convertido a baixas temperaturas, é promissora do ponto de vista da engenharia supramolecular, em que a combinação de técnica e de novos materiais pode produzir estruturas com propriedades controladas.

\section{AGRADECIMENTOS}

Os autores agradecem o suporte financeiro da FAPESP, FAPEMIG e CNPq.

\section{REFERÊNCIAS}

1. Greenham, N. C. Em Quantum Theory of Solids; Greenham, N. C.; Friend, R. H., eds.; R. E. Peierls: Oxford, 1995, vol. 49, cap. 1.

2. Parker, I. D.; J. Appl. Phys. 1994, 75, 1656.

3. Bradley, D. D. C.; J. Phys. D: Appl. Phys. 1987, 20, 1389.

4. Ho, P. K. H.; Thomas, D. S.; Friend, R. H.; Tessler, N.; Science 1999, 285, 233.

5. Marletta, A.; Gonçalves, D.; Oliveira Jr., O. N.; Faria, R. M.; Guimarães, F. E. G.; Adv. Mater. 2000, 12, 69.

6. Yang, Y.; Heeger, A. J.; Appl. Phys. Lett. 1994, 64, 1245.

7. Travain, S. A.; Libardi, L. H.; Marletta, A.; Giacometti, J. A.; Guimarães, F. E. G.; Faria, R. M.; Mol. Cryst. Liq. Cryst. Sci. Technol. 2002, 374, 439.

8. Stejskal, J.; Sapurina, I.; Prokes, J.; Zemek, J.; Synth. Met. 1999, 105, 195.

9. Epstein, A. J.; MacDiarmid, A. G.; Mol. Cryst. Liq. Cryst. Sci. Technol. 1988, 160, 165.

10. Mattoso, L. H. C.; Faria, R. M.; Bulhões, L. O. S.; Mac Diarmid, A. G.; J. Polym. Sci., Part A: Polym. Chem. 1994, 32, 2147.

11. Decher, G.; Hong, J. D.; Schmitt, J.; Thin Solid Films 1992, 210/211, 831.

12. Raposo, M.; Pontes, R. S.; Mattoso, L. H. C.; Oliveira Jr., O. N.; Macromolecules 1997, 30, 6095.

13. Pontes, R. S.; Raposo, M.; Camilo, C. S.; Dhanabalan, A.; Ferreira, M.; Oliveira Jr., O. N.; Phys. Status Solidi A 1999, 173, 41

14. Li, W.; Wan, M.; J. Appl. Polym. Sci. 1999, 71, 615.

15. Wan, M.; J. Polym. Sci., Part A: Polym. Chem. 1992, 30, 543. 